\title{
Global Bridges: Development and Analysis of a Tobacco Treatment Network
}

\author{
Scott J. Leischow, ${ }^{1}$ J. Taylor Hays, ${ }^{2}$ Thomas J. Glynn, ${ }^{3}$ Katherine E. Kemper, ${ }^{1}$ Janet Okamoto, ${ }^{2}$ \\ and Richard D. Hurt ${ }^{1}$ \\ ${ }^{1}$ Mayo Clinic, Scottsdale, AZ, USA \\ 2 Mayo Clinic, Rochester, $M N, A Z$ \\ ${ }^{3}$ Stanford University, Palo Alto, CA, USA
}

\begin{abstract}
$\mathrm{T}$ he Framework Convention on Tobacco Control (FCTC) set standards for global tobacco control, including the implementation of evidence-based tobacco dependence treatment. However, efforts to implement tobacco treatment programmes globally have been few. In order to expand tobacco treatment expertise and programmes, a new network called Global Bridges (GB) was established. This network provided training in tobacco treatment and opportunities to share best practices on implementation of tobacco dependence treatment and training programmes. In this analysis of the GB network, we found that $75 \%$ of the network members attended trainings, $60 \%$ disseminated knowledge gained through GB training, and network centralization was high (0.85). These results demonstrate initial success in network implementation, and create a foundation for expanded focus on tobacco treatment globally.
\end{abstract}

Keywords: tobacco treatment, network analysis, global, low and middle income country

\section{Introduction}

Tobacco use is recognised as the greatest preventable cause of death and disability in the world because it kills more than 6 million people every year, including more than 600,000 non-smokers who die from exposure to tobacco smoke (WHO, 2013a). At present, there are over 1 billion smokers in the world, and almost $80 \%$ of them live in low- and middle-income countries (LMIC) where tobacco-caused morbidity and mortality is high (WHO, 2013a). The World Health Organization (WHO) projects that the annual death toll attributable to tobacco will rise to over 8 million people by 2030 unless decisive action is taken (WHO, 2013a). The WHO FCTC, the world's first global health treaty, which has been ratified by 180 countries, covering $90 \%$ of the world's population, provides a blueprint for productive action (WHO, 2013b). The FCTC includes a specific provision on treatment (Article 14), as well as other evidence-based tobacco control policies, that motivate smokers to seek treatment, such as smoke-free workplaces, taxation, warning labels, and advertising bans (WHO, 2013b). In many countries that are parties to the FCTC, particularly in LMICs, implementa- tion of the treatment provisions has lagged behind other policies even though treatment for tobacco dependence is efficacious and cost effective (US DHHS, 2014).

However, especially in LMICs, healthcare professionals (HCPs) often lack experience and knowledge about the devastating health toll of tobacco and how to effectively treat tobacco dependence. Building capacity for tobacco dependence treatment among HCPs remains an unaddressed need in most countries (Raw and Murray, 2012). In addition to treatment capacity, credible health advocacy is urgently needed in countries where tobacco represents one of the most important threats to public health. Because HCPs are among the most educated members of any community and are viewed as the most credible source of health information, they represent a highly motivated yet largely untapped force for addressing the tobacco pandemic (WHO, 2005).

Recognising this unfulfilled yet urgent need, Pfizer created the Global Healthcare Alliance (GHA), a nonbranded initiative, in 2006. The focus of the GHA was to bring together, in a series of annual meetings, HCPs who were interested in promoting tobacco dependence 
treatment at the local and national levels. Over several years, the GHA built momentum and enthusiasm among participants worldwide. Eventually, Pfizer decided to transition out of its role of coordinating the GHA, and Mayo Clinic submitted a proposal to Pfizer Medical Education Group to extend this work. The grant was approved in mid-2010, and a new, independent initiative called GB was created with administrative leadership based at the Mayo Clinic in Rochester, Minnesota (Hurt and Hays) with collaborative leaders at the University of Arizona (Leischow, who eventually moved to the Mayo Clinic in Arizona) and American Cancer Society (Glynn). Since that time, the GB leadership team has dramatically expanded the scope, reach, and impact from the original GHA.

While the original GHA was an important initiative, its focal point was an annual meeting with no formal interaction or networking between those meetings. GB set out to ensure more continuous communication between network members. GBs' mission is to: 'Create and mobilize a global network of HCPs and organizations dedicated to advancing effective tobacco dependence treatment and advocating for effective tobacco control policy'. Such policies can impact treatment either directly or indirectly (e.g. smoke-free workplaces, health insurance coverage). While primary beneficiaries of the GB mission are HCPs and their patients, society as a whole ultimately benefits from a reduction in tobacco use.

Given that broad mission, and in addition to assuring that the initiative itself would be sufficiently funded, the GB leadership team developed the following specific objectives - each of which was intended to function synergistically with the other:

- Build a global HCP network and create opportunities to share tobacco dependence treatment and advocacy expertise among network members within and across regions.

- Provide state-of-the-science training in evidence-based pharmacological and behavioural treatment and advocacy for network members.

- Facilitate the implementation of Article 14 of the FCTC in every nation.

The premise behind developing a network of HCPs and organisations that are dedicated to tobacco addiction treatment, and building into that network, an infrastructure intended to foster sharing approaches that work locally, is an outgrowth of our work on NCI Monograph 18 (NCI, 2008; Leischow et al., 2008). One of the objectives of that Monograph was to encourage thinking about tobacco control from a 'systems' perspective, including to 'develop and optimize networks to enable the community to more efficiently address varied populations, critical channels for intervention, and intervention types'. Since that seminal Monograph was published, we have investigated multiple networks to better understand how network structure and function can be optimised to improve tobacco con- trol decision-making (Bonito et al., 2013; Harris et al., 2012a; 2012b; Leischow et al., 2010; 2012; Provan, Beagles, Leischow, \& Mercken, 2013; Provan, Leischow, Keagy, \& Nodora, 2009; Saul, Bonito, Provan, Ruppel, \& Leischow, 2014; Terpstra et al, 2013). By creating a global tobacco treatment network, fostering communication and collaboration in that network, and then using network analyses to identify ways to strengthen and expand the network, our goal was to create and sustain an infrastructure that could eventually lead to global improvements in tobacco treatment.

In a short period of time, the GB leadership team established a robust and growing international network of HCPs focused on tobacco dependence training and advocacy. In a tobacco control world in which treatment is considered a low priority by most countries (Raw and Murray, 2012), GB is now a highly visible independent global voice on tobacco dependence treatment. In order to better understand the current state of the GB network, we have begun the process of analysing that network in order to better understand its structure and function in order to expand and strengthen it. This paper describes our approach to building and analysing this unique network.

\section{Methods}

\section{Creation of Global Bridges as a Training and Education Network}

In order to begin developing the tobacco treatment network, the GB leadership team identified Regional Leaders and established regional partnerships in four of six WHO regions in 2010: Africa, the Middle East, Latin America, and the Caribbean, and, to a far lesser extent, Europe. Those Regional Leaders were identified by GB leadership as individuals who were active in their region regarding tobacco treatment, were active in collaborating across regions in tobacco treatment, and had the interest and capacity to build a network in their region. The European GB network began later than the other three, and had somewhat different characteristics because considerably more tobacco treatment infrastructure existed in Europe before GB began. Other regions (e.g. North America) were not included in the beginning of the initiative because resources for the project implementation were limited. Each Regional Leader assembled an advisory group comprised of expert leaders from within their region, and rapidly began implementing training courses and programmes in multiple locations within their respective regions. Those who attended any training or programme were asked if they wished to join the GB network. We collected email addresses of those who indicated interest so that we could follow up to determine whether the training had any impact on their efforts. At the time of survey implementation in 2013, GB courses and trainings meetings achieved a combined attendance of more than 22,000 people from more than 50 countries.

In addition, a multilingual website (globalbridges.org) was established to foster a worldwide information and 
education platform and forum for collaboration among colleagues. Content on the website is provided by GB regional leaders and other network members, and the website offers resources, blogs, and member spotlights in seven languages. In addition, a listserv open to GB members was implemented to foster knowledge sharing globally.

GB leadership also initiated ongoing collaborations with leading tobacco control/treatment organisations, including the Framework Convention Alliance, Association for Treatment of Tobacco Use and Dependence (ATTUD), Society for Research on Nicotine and Tobacco (SRNT), Tobacco Control Nurses International, Global Smokefree Partnership, University of Toronto Centre for Addiction and Mental Health (CAMH), World Heart Federation, the Hong Kong Department of Health, Hong Kong Hospital Authority, and others. Those organisations were encouraged to ask their members to also become members of GB.

As a result of the trainings, courses, website, blog, listserv, and collaborations with other organisations, over 2,000 people from 85 countries have become members of the GB network.

\section{Network Analysis Rationale and Methods}

In order to understand whether the GB network truly was functioning as a knowledge-sharing network, we developed and implemented an online survey of members in order to characterise the network and better understand how members communicate and interact with each other. More specifically, our goals with the survey were to address the following questions: (a) Did GB actually create a network that was measurable? (b) If the network was measurable, would it be possible to assess the nature of interactions within and between regions to assess the nature of the network? And (c), within each region, was there evidence of knowledge sharing suggesting that GB was assessing and fostering leadership beyond the leadership group funded to implement the GB network?

Survey description. The GB leadership developed a 44item survey instrument to assess communication between members of the GB network in order to examine whether the primary goals of the initiative were being met. In addition to basic demographic information, examples of questions asked of each respondent included the following: (a) type of professional position and duration in that position, (b) organisation description, including services offered, (c) GB involvement, including attendance at regional GB trainings and top three individuals or organisations in the GB network you interact with the most, and (d) top three individuals or organisations you've collaborated with about tobacco dependence treatment. The questions were pilot tested by the GB leadership team, including the leaders of each region, and were translated and back translated in Spanish to ensure consistency and accuracy. Previous GB website usage data indicated that the predominant languages of the GB website users were English and Spanish.

Implementation. E-mail solicitations, including a link to the online survey, were sent in March 2013 to 526 members who had registered through the GB website and provided an email address. The survey remained open in March and April 2013 with weekly reminder e-mails. In addition, the regional GB partners disseminated the survey link through their listservs and encouraged participation. A total of 233 responses were received online for a response rate of $44 \%$.

Data management and analysis. Data was downloaded from the online survey instrument. Data cleaning, organization, and descriptive statistics were calculated using Stata 11 (Statacorp, 2009). Basic network metrics were calculated using UCINet 6 (Borgatti, Everett, \& Freeman, 2002). Visualisations were done using the Gephi software for network analysis (Bastian, Heymann, \& Jacomy, 2009) and UCINet 6. Organisation names were maintained and presented in the network diagrams, while individual names were replaced by general role descriptors (e.g. Regional Director). For clarity, only the most prominent nodes or nodes that play a key role in the network are labelled in the diagrams. Respondents' countries were coded by WHO region. Reported ties, whether they were individuals or organisations, were also coded by WHO region. The analysis included both individuals and organisations as nodes in the network as the survey questions asked respondents to name either individuals or organisations. No aggregation was made to the organisational level, so individuals were represented in the analysis as an individual rather than a representative of their organisations. Organisations only appear as nodes if respondents named an organisation (e.g. SRNT).

\section{Results \\ Response Characteristics}

Because the European GB network began later, fewer people registered in with the GB website and the network in the European region appears less connected with fewer nodes represented. $53 \%$ of the respondents were from the Americas, with the majority from the Americas region, $40 \%$ of total respondents, coming from South and Central America. 20\% of the responses were from Africa, 14\% from the Middle East, and 8\% from Europe. Only 5\% of responses were from Asian countries, a region where GB had only preliminary discussions with those involved in tobacco dependence treatment, with the exception of Hong Kong where there is an extensive collaboration with the Department of Health and Hospital Authority. The low number of responses from Europe and Asia reflected the minimal involvement of GB in those regions early this initiative, and the lack of involvement in the network by tobacco control organisations in Asia, though some individual members of GB were located in Asian countries.

Survey respondents were closely split by gender $(56 \%$ male, $44 \%$ female). $27 \%$ were physicians, $15 \%$ were 
non-physician clinicians (e.g. nurses, psychologists, etc.), $13 \%$ were executive leaders, and $13 \%$ were programme managers. The remaining 32\% reported other professions such as civil servants, health educators, and researchers. The majority of survey respondents worked for an organisation/institution that provided treatment or other clinical services (65\%) and education/outreach (52\%). Research $(44 \%)$, policy $(34 \%)$, and technical training $(34 \%)$ were also common services provided by participants' organisations, most of which provided more than one type of service. $75 \%$ of the respondents reported that they had attended a GB training, with an average attendance of $1.43(\mathrm{SD}=2.6) \mathrm{GB}$ events. The majority of respondents reported that they had collaborated with an organisation/individual on tobacco dependence treatment (74\%) and $40 \%$ reported they had interacted with other individuals or organisations that are members of the GB network. Finally, $60.3 \%$ reported sharing information they received through GB involvement with other individuals and/or organisations.

\section{Network Measures}

(Figure 1) shows the global map of self-reported interactions with other GB members. This figure shows there is an established network of GB collaborators that span across regions and groups. The 'nodes' in the figure are shaded by betweenness centrality and sized by in-degree centrality.

Overall network centralisation is high for the GB interaction network (0.85). This measure, calculated at the network level, indicates that the network is centralised around a few key nodes. This indicates a likely dependence on the GB Executive Committee and the Regional Directors as key points of contact and connection among the different regions, as indicated by the fact that these individuals has both high in-degree centrality (a measure of the number of incoming ties to a node) as well as betweenness centrality (a measure that indicates the degree to which a node connects otherwise unconnected nodes in the network). (Table 1) reports a summary of these network measures for the GB interaction network. In addition, both the South American and African regions have additional nodes, besides the Regional Directors, with high in-degree centrality that are prominent within the region. In South America, an internationally recognised leader in tobacco control (who is not the Regional Director in $\mathrm{GB}$ ) is seen as a large darker gray node to the right of the Regional Director. This individual had some involvement with the GB network as a trainer (as noted by the tag in the figure). In Africa, there is an active group in Nigeria that is connected to the Regional Director, but who also seem to be developing their own regional network within the Africa region. In the Middle East, the Regional Director is the largest node, and the nodes in this region appear to be connected through this node. Because the GB effort in Europe began later than the others, no major central nodes exist. The darker nodes in (Figure 1) indi- cate that the individual connects otherwise unconnected nodes. These two measures of node centrality, in-degree and betweenness, are typically highly correlated as can be seen by the darker nodes also being larger in size. However, not all nodes with a lot of incoming ties have high degree centrality. The nodes with both high in-degree and betweenness centrality likely serve as the key individuals that connect the most individuals and groups in the network.

(Figure 2) depicts the tobacco dependence treatment contact and collaboration network. In this Figure, the different node shapes represent different regions and nodes are sized by in-degree centrality. Like the GB interaction network shown in (Figure 1), network centralization is also high in the tobacco treatment communication network (0.87). This network is derived from respondents who indicated that they communicated with individuals or organisations regarding tobacco dependence treatment. Unlike the GB interaction network, however, in this network respondents' named more organisations than individuals. The organisations that appear the most prominent in the tobacco dependence treatment network (Figure 2) have been influenced by GB activity and include mostly organisations that GB has collaborated with at the regional level for trainings and other activities (e.g. the Ethiopian Public Health Association, ATTUD, and Health Authority Abu Dhabi). This network data for tobacco dependence treatment collaborations also shows the beginnings of connections across countries and regions. While the majority of the nodes were around the periphery of the network, (Figure 2) demonstrates a clear core to this network spanning across regions, with ATTUD and Mayo Clinic (where the GB efforts originated) serving as the most prominent nodes in this core. However, each region is represented in this collaboration network, with organisations included for many regions and countries that are serving as regional resources for individuals working in tobacco treatment.

\section{Discussion and Conclusions}

The FCTC has identified tobacco treatment as a priority (WHO, 2013b), yet few efforts have been implemented globally to foster communication and collaboration on ways to maximise and optimise the treatment of tobacco addiction (Raw and Murray, 2012). The SRNT has done outstanding work compiling evidence-based approaches for tobacco treatment, and organising that information on a website (www.treattobacco.net) in multiple languages so that in-country tobacco treatment champions have the tools needed to implement tobacco treatment programmes. But treattobacco.net is not designed to help those who wish to implement programmes. Thus, the creation of a network that allows those who are interested in tobacco treatment programme implementation to share approaches that work within specific countries or regions, to explore strategies on how to work through programme implementation barriers, and to champion tobacco 


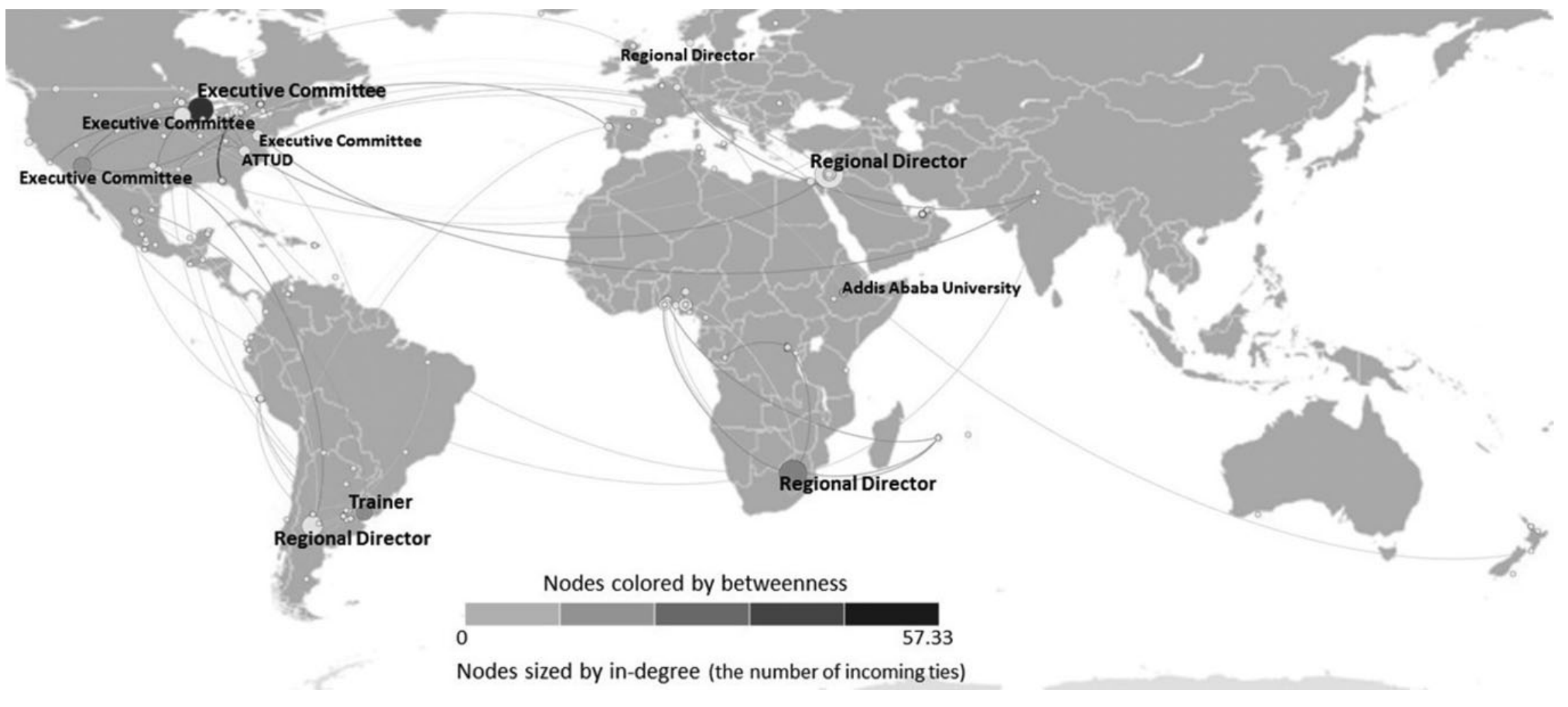

Figure 1

Network map of global bridges interaction and collaboration. 


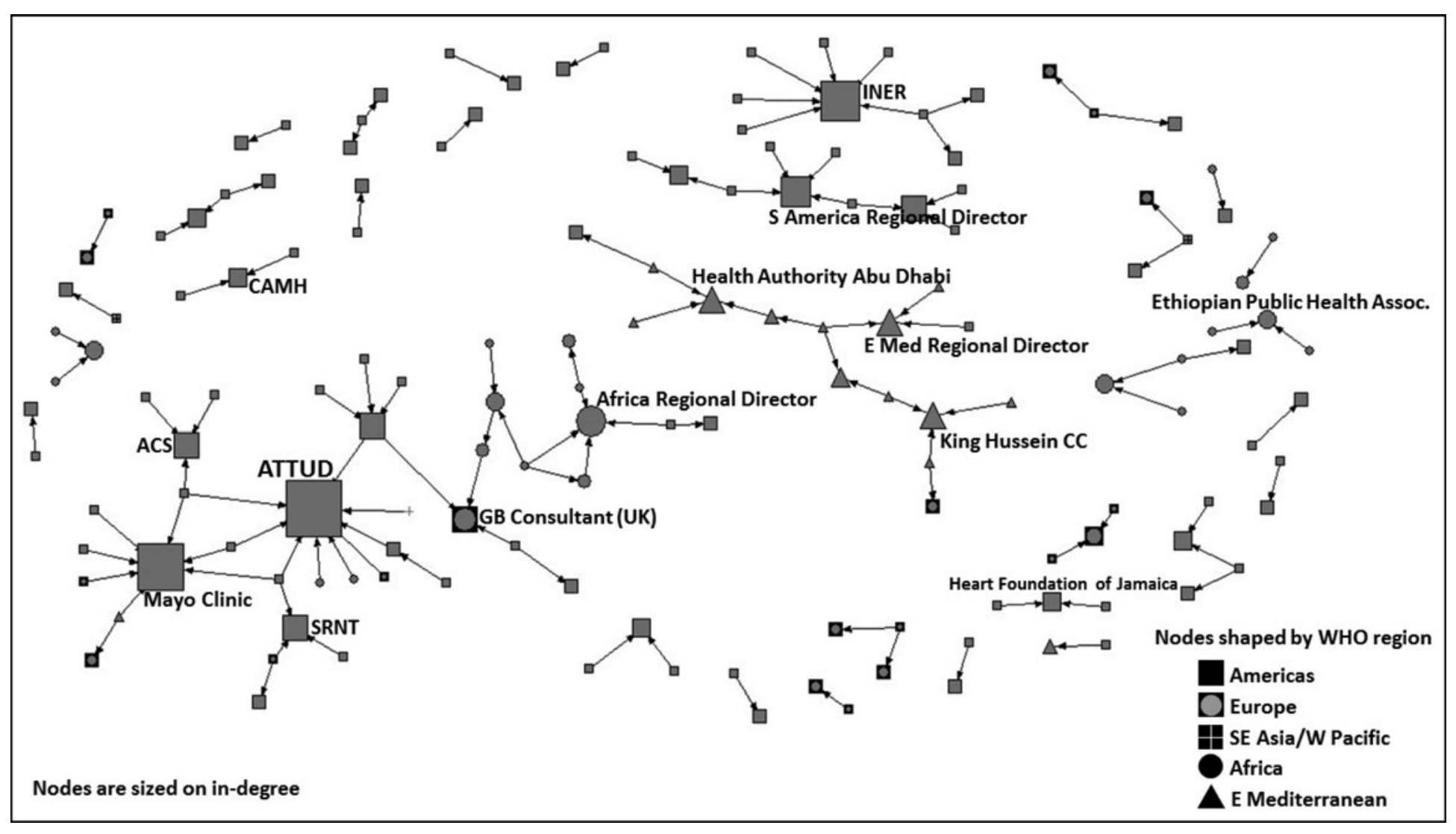

Figure 2

Communication and collaboration on tobacco dependence treatment. 


\begin{tabular}{|c|c|}
\hline \multicolumn{2}{|l|}{$\begin{array}{l}\text { Table } 1 \\
\text { Global bridges interaction network measures summary }\end{array}$} \\
\hline \multicolumn{2}{|l|}{ \# of Nodes } \\
\hline Total & 162 \\
\hline Africa & 35 \\
\hline Americas & 73 \\
\hline Eastern Mediterranean & 26 \\
\hline Europe & 22 \\
\hline South-East Asia \& Western Pacific & 6 \\
\hline \multicolumn{2}{|l|}{ \# of Ties } \\
\hline Total & 163 \\
\hline Africa & 37 \\
\hline Americas & 75 \\
\hline Eastern Mediterranean & 30 \\
\hline Europe & 17 \\
\hline South-East Asia \& Western Pacific & 4 \\
\hline Network Centralization & 0.85 \\
\hline Mean Betweenness Centrality & $0.562(4.27)$ \\
\hline Mean In-Degree Centrality & $1.07(2.23)$ \\
\hline Nodes with highest in-degree & \# incoming ties \\
\hline GB Executive Committee Member (GB Executive Director) & 22 \\
\hline Africa Regional Director & 21 \\
\hline E. Mediterranean Regional Director & 20 \\
\hline GB Executive Committee Member & 14 \\
\hline S. America Regional Director & 13 \\
\hline
\end{tabular}

treatment as a priority has great potential value. The core question we sought to address is whether the creation of such a network would facilitate communication and collaboration, since just building a network does not assure that it will be used. Fortunately, the results of this first analysis demonstrates that it can and is being used.

Our work building and analysing a new tobacco treatment network has relevance in several ways. First, understanding the processes and evolution of building an international tobacco control network is timely and relevant as attention is increasingly turned towards LMIC. Most higher income countries, e.g. in the United States and western Europe, have made great advances in providing tobacco treatment options to smokers. While improvements are needed even in how high-income countries provide treatment, the infrastructure in those countries is generally much greater than in LMIC (Raw and Murray, 2012). By creating a network to share ideas on what works and what doesn't, particularly within specific regions that have unique needs and characteristics, GB has begun the process of helping those are dedicated to tobacco treatment to learn what others are doing to achieve the FCTC Article 14 objectives. For example, there are unique cultural, religious, and language circumstances in the Middle East that must be considered when developing and implementing tobacco treatment programmes.

Second, our network survey responses show activity and ties directly resulting from participation in GB activities and training. GB sought to identify and engage opinion leaders in tobacco treatment within each region given the data showing that doing so has the greatest potential to speed diffusion of innovations within a network (Valente \& Davis, 1999), and our data provide an early suggestion that this approach fosters the ties needed to improve diffusion of tobacco treatment. The connections within most regions where GB has been implemented are not broad or extensive yet, but it is clear that communication is occurring. While such communication may seem trivial, when creating a new network, it is essential to demonstrate such communication to justify continued effort at building and sustaining the network, and it is valuable to assess success in programme implementation (Valente, 2012; Valente, Palinkas, Czaja, Chu, \& Brown, 2015). The tobacco dependence treatment collaboration network data also indicated that professional and organisations ties are forming through GB activities and efforts, and that the GB network is influencing contact and introducing resources concerning tobacco dependence treatment to its members. 
Some limitations should be mentioned related to this analysis. Due to the early stage of development of this network and the length of time needed to grow a strongly connected and maximally effective network of collaborators, the network data was limited. The response rate was respectable for an email solicited survey, but the lack of data for the full network did pose some limitations on the analysis. For example, many common network metrics were not informative for this analysis due to the sparse nature of the tie data. Network density and reciprocity very low and we were unable to tease out whether or not this was a feature of the GB network or lack of data. Also, the open-ended survey questions used to generate the network data were limited to three named collaborators for each type of network question. While this is common practice in order to decrease respondent burden, it also limits degree centrality measures. In particular, outdegree centrality was not used as metric in the analysis as almost all respondents have the maximum number of three outgoing ties. This also was not a 'bounded' network in the traditional sense, in that we did not have a full roster of all network members to be able to compare survey responses to. The registration data of $526 \mathrm{~GB}$ members used to contact potential respondents and calculate the response rate did not include specific name information, only email addresses. The GB network is also known to be larger than 526, as mentioned above, but there is no tracking or contact information for all attendees of GB trainings, which is a limitations as the full nature of the network is not currently measurable. Finally, response rates were likely limited by the survey languages, as the survey was only available in English and Spanish. While most GB training were conducted at least in part, in English, we know that responses were limited by those who did not read English or Spanish well enough to complete the survey. Despite these known limitations, the analysis presented here still provides a valuable look at a growing network of professionals working in the area of global tobacco dependence treatment and will serve to inform future efforts to continue to grow the knowledge-base and collaborations to ultimately work towards decreasing the burden of tobacco-related disease worldwide.

Because GB is a new network, and one built around regional leaders, it is clearly very centralised. This is expected, and very likely essential, because it assures that the Executive Committee and the Regional Directors within each region function as 'champions' for tobacco treatment. As the network matures, the network should become less centralised, or less structured around the Executive Committee and Regional Directors, as the capacity and level of tobacco treatment expertise in each region increases. The GB leadership has already begun the process of encouraging more reliance on regional leaders and collaborators. Evidence that this has already begun can be seen in this analysis, such as the growth of regional connections around Nigeria in a different part of Africa from the Regional Director. Continuation funding for the network has a very different structure that will hopefully help to encourage regional growth that will contribute to less centralised network and strengthen network ties at the local level. Future network analyses will demonstrate whether or not the goal of developing a broader and less centralised network will have been achieved.

\section{Acknowledgements}

We are grateful for the leadership, collaboration and significant effort provided by the leaders of the Global Bridges regional leaders.

\section{Financial Support}

Pfizer, Inc.; American Cancer Society

\section{Conflict of Interest}

SJL: research funding from McNeil Consumer; JTH: research funding from Pfizer; TJG: educational grant from Pfizer Independent Grants for Learning and Change Clinic; KEK: educational grant from Pfizer Independent Grants for Learning and Change; JO: nothing to disclose; RDH: scientific ad board for Chrono Therapeutics.

\section{Ethical Standards}

The authors assert that all procedures contributing to this work comply with the ethical standards of the relevant national and institutional committees on human experimentation and with the Helsinki Declaration of 1975, as revised in 2008.

\section{References}

Bastian, M., Heymann, S., \& Jacomy, M. (2009). Gephi: An open source software for exploring and manipulating networks. International AAAI Conference on Weblogs and Social Media.

Bonito, J., Ruppel, E., Saul, J., \& Leischow, S. J. (2013). Assessing the preconditions for communication influence on organizational decision making: The North American quitline consortium. Health Communications, 28(3), 248-259. DOI:10.1080/10410236.2012.673245.

Borgatti, S. P., Everett, M. G., \& Freeman, L. C. (2002). Ucinet 6 for windows: Software for social network analysis. Harvard, MA: Analytic Technologies.

Harris, J. K., Carothers, B. J., Wald, L. M., Shelton, S. C., \& Leischow, S. J. (2012b). Interpersonal influence among tobacco control professionals in the United States department of health and human services. Journal of Public Health Research, 1(1), 67-74.

Harris, J. K., Provan, K. G., Johnson, K. J., \& Leischow, S. J. (2012a). Drawbacks and benefits associated with inter-organizational collaboration along the discoverydevelopment-delivery continuum: A cancer research network case study. Implementation Science, 7, 69. doi:10.1186/1748-5908-7-69.

Leischow, S. J., Best, A., Trochim, W. M., Clark, P. I., Gallagher, R. S., Marcus, S. E. et al. (2008). Systems thinking to improve 
the public's health. American Journal of Preventive Medicine, 35(2), S196-S203.

Leischow, S. J., Luke, D. A., Mueller, N., Harris, J. K., Ponder, P., Marcus, S. et al. (2010). Mapping U.S. government tobacco control leadership: Networked for success?. Nicotine and Tobacco Research, 12(9), 888-894.

Leischow, S. J., Provan, K., Beagles, J., Bonito, J., Ruppel, E., Moor, M. et al. (2012). Mapping tobacco quitlines in North America: Signaling pathways to improve treatment. American Journal of Public Health, 102(11), 2123-2128. doi: 10.2105/AJPH.2011.300529.

Provan, K. G., Leischow, S. J., Keagy, J., \& Nodora, J. (2009). Research collaboration in the discovery, development, and delivery networks of a statewide comprehensive cancer coalition. Evaluation and Program Planning,33(4), 349-55. Epub 2009.

Provan, K. G., Beagles, J., Leischow, S. J., \& Mercken, L. (2013). Awareness of evidence-based practices by organizations in a smoking cessation network. Journal of $P u b$ lic Administration Research and Theory, 23(1), 133-15. doi: 10.1093/jopart/mus011.

Raw, M., \& Murray, R. (Eds.) (2012). Tobacco watch. Implementation of FCTC article 14. Geneva, Framework Convention Alliance.

Saul, J. E., Bonito, J. A., Provan, K., Ruppel, E., \& Leischow, S. J. (2014). Implementation of tobacco cessation quitline practices in the United States and Canada. American Journal Public Health, 104(10), e98-105. doi: 10.2105/ AJPH.2014.302074.

StataCorp. (2009). Stata Statistical Software: Release 11. College Station, TX: StataCorp LP.

Terpstra, J., Best, A., Saul, J., \& Leischow, S. J. (2013). The complexity of institutionalizing evaluation as a best practice in
North American quitlines. American Journal of Evaluation, 34(3), 356-371. doi: 10.1177/1098214013487426.

U.S. Department of Health and Human Services. The Health Consequences of Smoking - 50 Years of Progress: A Report of the Surgeon General. Atlanta, GA: U.S. Department of Health and Human Services, Centers for Disease Control and Prevention, National Center for Chronic Disease Prevention and Health Promotion, Office on Smoking and Health, 2014.

Valente, T. W. (2012). Network interventions. Science, 337(49), 49-53. DOI: 10.1126/science.1217330.

Valente, T. W., \& Davis, R. L. (1999). Accelerating the diffusion of innovations using opinion leaders. The ANNALS of the American Academy of Political and Social Science, 566(1), 55-67. DOI: 10.1177/000271629956600105.

Valente, T. W., Palinkas, L. A., Czaja, S., Chu, K.-H., \& Brown, C. H. (2015) Social network analysis for program implementation. PLoS ONE, 10(6), e0131712. doi:10.1371/journal.pone.0131712.

World Bank. (1999). Curbing the epidemic: Governments and the economics of tobacco control. Tob. Control., 8(2), 196201.

World Health Organization. WHO Tobacco Free Initiative. The role of health professionals in tobacco control. WHO Press, World Health Organization, Geneva, Switzerland, 2005; http://www.who.int/tobacco/resources/publications/wntd/ 2005/bookletfinal_20april.pdf. Accessed January 6, 2016.

World Health Organization. (2013a). Media centre, tobacco, factsheets. Available at: http://www.who.int/mediacentre/ factsheets/fs339/en/. Accessed May 17, 2013.

World Health Organization.(2013b) Framework Convention on Tobacco Control. Guidelines for implementation of Article 14. November 2010; http://www.who.int/fctc/protocol/ guidelines/adopted/article_14/en/. Accessed May 17, 2013. 